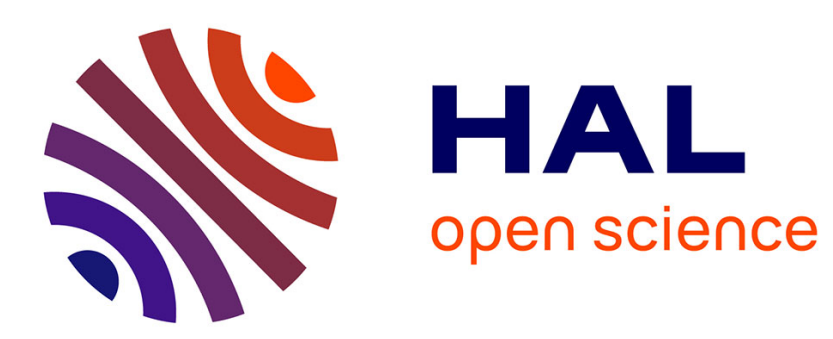

\title{
RECENT THEORETICAL AND EXPERIMENTAL ADVANCES IN THE UNDERSTANDING OF GRAIN BOUNDARY MIGRATION
}

Ch. Bauer

\section{- To cite this version:}

Ch. Bauer. RECENT THEORETICAL AND EXPERIMENTAL ADVANCES IN THE UNDERSTANDING OF GRAIN BOUNDARY MIGRATION. Journal de Physique Colloques, 1982, 43 (C6), pp.C6-187-C6-197. 10.1051/jphyscol:1982618 . jpa-00222299

\section{HAL Id: jpa-00222299 \\ https://hal.science/jpa-00222299}

Submitted on 1 Jan 1982

HAL is a multi-disciplinary open access archive for the deposit and dissemination of scientific research documents, whether they are published or not. The documents may come from teaching and research institutions in France or abroad, or from public or private research centers.
L'archive ouverte pluridisciplinaire HAL, est destinée au dépôt et à la diffusion de documents scientifiques de niveau recherche, publiés ou non, émanant des établissements d'enseignement et de recherche français ou étrangers, des laboratoires publics ou privés. 


\title{
RECENT THEORETICAL AND EXPERIMENTAL ADVANCES IN THE UNDERSTANDING OF GRAIN BOUNDARY MIGRATION
}

\author{
Ch. L. Bauer \\ Department of Metallurgical Engineering \& Materials Science, Camegie-Melzon \\ University, Pittsburgh, Pennsylvania, U.S.A.
}

\begin{abstract}
Résumé. - La migration des joints de grains continue à attirer beaucoup d'attention par son influence sur plusieurs procédés industriels importants. Le but de cet article et de faire un compte rendu des récents progrés théoriques et experimentaux qui aident à la comprêhension de ce phénomène afin de préciser 1 'état actuel des connaissances et d'identifier les problèmes qui demandent des études supplementaires.
\end{abstract}

Abstract. - The subject of grain boundary migration continues to command significant attention because of its relevance to many important technological processes. The purpose of this article is to review recent theoretical and experimental advances in the understanding of grain boundary migration in order to define the current state of present knowledge and identify important problems which require further investigation.

\section{Introduction}

The subject of grain boundary migration continues to command considerable attention because it represents the principal way in which a single phase material is transformed into a more perfect material of the same overall composition through systematic movement of an interface. The extent of such migration controls grain size, preferred orientation and redistribution of chemical impurities, all of which strongly affect many engineering properties of materials. The rate of migration is dependent on several important variables: First, it is strongly dependent on the rate of transferral of individual or clusters of atoms across the boundary, which, in turn, is strongly dependent on temperature and sensitive to detailed atomic structure of the boundary. Second, the rate is dependent on the driving force per unit boundary area, which is just the volume free energy change produced by passage of the boundary. These rather fundamental features of grain boundary motion, unfortunately, are immensely complicated by the fact that the grain boundary itself represents a plane imperfection and, therefore, may interact with other lattice imperfections and/or chemical impurities. Attempts to qualify such behavior have met with uneven success, mainly because theoretical models tend to focus on continuum or crystallographic features in regimes where imperfections and/or chemical impurities may or may not play a dominant role. Complete understanding of grain boundary migration is complicated further by the myriad of existing experimental results, often obtained under imprecise control of important variables, such as driving force and chemical. composition, and imprecise knowledge of grain boundary structure. This situation is also compounded by the fact that many important features of grain boundary migration occur on a scale too fine to be observed by conventional optical resolution.

The purpose of this article is to review recent theoretical and experimental advances in the understanding of grain boundary migration in order to define the current state of knowledge and to identify important problems which require further investigation. Moreover, attention is focused on fundamental aspects of grain boundary migration rather than on complex phenomena, such as discontinuous precipitation, interphase boundary migration, recrystallization and grain growth, although such phenomena may be mentioned when necessary to highlight certain fundamental aspects of grain boundary migration, at least within the space limitations of this article. Further details are reviewed in prior articles by Hofmann and Haessner, 1 Simpson, Winegard and Aust, 2 and Gleiter and Chalmers. 3 
The remainder of this article is divided into several sections: First, relevant theoretical background is presented, then experimental techniques are reviewed briefly and recent experimental results are examined and compared with existing theoretical predictions. Finally, the present state of understanding of grain boundary migration is summarized in the concluding section of this article.

\section{Theoretical Background}

The kinetics of grain boundary migration are usually described by relating macroscopic grain boundary velocity $v$ to a given impurity concentration $c$ and applied driving force $F$. Functionally, $v$ may be related to $F$ by the expression

$$
\mathrm{v}=\mathrm{MF} \text {, }
$$

where $M$ denotes macroscopic grain boundary mobility. In turn, by a simple force balance, $M$ may be resolved into an intrinsic component $M_{I}$ and an extrinsic component $M_{I I}$, which are related to $M$ by the expression

$$
1 / M=1 / M_{I}+1 / M_{I I} \text {. }
$$

Equations 1 and 2 merely acknowledge the fact that grain boundary mobility is a complicated function of grain boundary velocity and is equivalent to the expression

$$
\mathrm{v}=\mathrm{MF}^{\mathrm{m}} \text {. }
$$

Equation 3, however, is somewhat artificial, since the correct driving force is adjusted to compensate for actual variations in grain boundary mobility, at least for the general case of Newtonian behavior, where displacement is proportional to applied force.

The quantity $M_{T}$ is associated with transfer of matrix atoms from one grain to another by a diffusional process and is given by the general expression

$$
M_{I}=M_{I O} \exp (-Q / k T) \text {, }
$$

where $M_{10}$ denotes a temperature independent mobility, $Q$ denotes an activation energy for transferral of matrix atoms from one side of the boundary to the other, $k$ denotes Boltzmann's constant, and $T$ denotes absolute temperature. In contrast to $M_{T}$, which is independent of impurity concentration, $M_{T T}$ is usually a complicated function of $T$, $c$ and $F$. It is recognized that the great variety of existing experimental results is generalily due to the sensitive manner by which $M$ depends on $c$ and other such extrinsic variables. Consequently, it is important to understand processes affecting $M$, and thereby gain further insight into the complex phenomenon of grain boundary migration.

A second fundamental parameter in Eq. 1 is the driving force or pressure acting on unit area of the boundary. The most common origins of driving force are due to a difference in free energy between adjacent grains defining a boundary and capillary effects. Still other forces may originate at the periphery of the boundary rather than as a true pressure. An example of this type of force occurs when a boundary intersects a free surface so that the surface energies on each side of the boundary are different. In this case, the boundary tends to migrate in such a manner so that the higher energy surface is consumed by the lower energy one. Such surface forces generally vary inversely with specimen thickness, since this force is transmitted to the entire boundary area. The theoretical limit for all driving forces may be estimated by assuming that the maximum free energy change occurs when an atom crosses the moving boundary, which, in turn, may be approximated by the heat of sublimation, or about $10^{10} \mathrm{dyn} / \mathrm{cm}^{2}$. This value of $\mathrm{F}$ is similar to the limiting value for the capillary driving force, which may be evaluated by assuming that grain boundary curvature is identical to the atomic radius. A complete survey of driving forces for grain boundary migration including origins, analytical expressions, and estimated magnitudes is presented elsewhere. ${ }^{4}$

The most successful theories of grain boundary migration to date have been advanced by Liucke and Detert ${ }^{5}, \mathrm{Cahn}^{6}$ and Liicke and Stiuwe. ${ }^{7,8}$ These theories are based on the concept of interaction of chemical impurities with a moving grain boundary. This interaction is characterized by an interaction energy $U(x)$, which varies 
with perpendicular distance $x$ from a planar grain boundary. As a result, an excess (or deficit) concentration of impurities may be associated with the boundary and described formally by an excess concentration profile, which is symetric with respect to boundary position for a stationary boundary, but becomes asymnetric when the boundary is in motion. This asymmetry produces a drag force on the boundary so that concomitant (extrinsic) grain boundary velocity is controlled by diffusion of impurities, characterized by diffusion coefficient $D(x)$, across the grain boundary. As velocity increases, excess impurity concentration first increases (due to greater increase of asymmetry), but then gradually decreases (due to greater decrease of net excess impurity concentration). At high velocities, the impurities can no longer exert sufficient drag force to counteract the driving force (the net force on the boundary must vanish in steady state) and breakaway of the boundary from impurities occurs. Thereafter, intrinsic grain boundary velocity is not appreciably affected by the presence of impurities.

By considering diffusion of impurities in the potential field of the grain boundary at constant velocity, the (excess) concentration profile can be expressed in terms of $U(x)$ and $D(x)$. The sum of all forces between excess impurities and the grain boundary represents the (net) drag force, which may be combined to yield the following approximate expression for extrinsic grain boundary mobility

$$
1 / \mathrm{M}_{\mathrm{II}}=\mathrm{Ac}_{\mathrm{o}} /\left(1+\mathrm{Bv}^{2}\right) \text {, }
$$

where $c_{0}$ denotes bulk impurity content of the specimen and $A$ and $B$ denote drag parameters, which are complex functions of $U(x), D(x)$ and $T$. (In physical terms, A represents reciprocal grain boundary mobility at unit impurity concentration and $1 / B$ represents the average drift velocity of impurities across the boundary.) For sufficiently low driving force $(M F<1 / B)$ or high solute concentration $\left(F / A c_{0}<1 / B\right)$, Eq. 5 reduces to the expression

$$
F / v=1 / M=1 / M_{I}+A c_{0} .
$$

Therefore, at low values of the driving force the velocity is dependent on the rate of solute atom transfer across the grain boundary, whereas at high values of the driving force, the velocity is independent of impurity content. In either extreme, the velocity is proportional to the driving force, but with entirely different values of the apparent mobility. A schematic plot of grain boundary velocity versus driving force for various values of $c_{0}$ is presented in Fig. 1, wherein the low and highvelocity (asymptotic) limits are given by straight lines. It should be noted that the limiting driving force for breakaway is independent of graln boundary velocity.

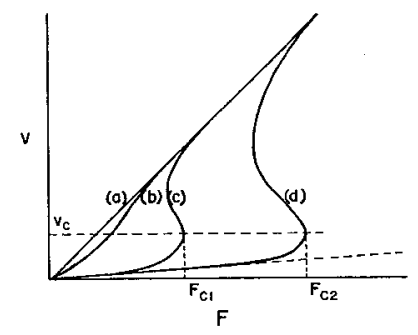

Fig. 1. Schematic representation of grain boundary velocity $\mathrm{v}$ as a function of driving force $F$ for (a) pure material, (b) impure material not exhibiting breakaway, (c) impure material exhibiting breakaway at $F_{c l}$, and (d) highly impure material exhibiting breakaway at $\mathrm{F}_{\mathrm{C}_{2}}$. Note that breakaway occurs at constant critical velocity $v_{C}$ and limiting slopes (mobilities) are given by $M=M_{T} /(1+$ $\mathrm{M}_{\mathrm{I}} \mathrm{Ac_{0 }}$ ), which reduce to $\mathrm{M}_{\mathrm{I}}$ and $1 / \mathrm{Ac}_{\mathrm{o}}$ for the intrinsic and extrinsic cases, respectively.

The aforementioned model may be refined by hypothesizing other forms for the drag force or differences in transferral rate of solute and solvent atoms across the boundary, but the principal qualitative features remain unchanged. All of these models, however, force the boundary to maintain a planar configuration and/or the excess impurity concentration to be independent of position along the boundary. These 
conditions do indeed obtain when grain boundary surface energy is infinite (forcing the boundary to be planar) or when the diffusion coefficient of impurities parallel to the boundary is infinite (instantaneously smoothing out fluctuations in the excess impurity concentration). For realistic values of material parameters, however, retention of these constraints is not justified and entirely new concepts must be introduced in order to provide a correct interpretation of grain boundary migration.

The aforementioned constraints have been relaxed by Roy and Bauer 9,10 , who report that, in general, a moving grain boundary under the influence of impurities tends to assume nonplanar configurations characterized by a nonuniform lateral distribution of impurities. Boundary shape may fluctuate greatly with only slight redistribution of impurities or the impurity profile may fluctuate greatly with only slight variation of boundary shape. Likelihood of these processes occurring depends on the relative degree of impurity diffusion parallel $D_{4}$ and perpendicular $D_{+}$to moving boundary. The extreme cases are illustrated in Fig. 2, wherein boundary displacement in a moving frame of reference $\Delta \xi(x, t)$ and concentration profile along the boundary $\Delta c(x, t)$ are given for the cases where $\beta D_{1}>\beta D_{n}$ and $D_{1}>\beta D_{\|}$respectively, and $\beta$ is a dimensionless parameter which is inversely proportional to both impurity concentration and absolute temperature.
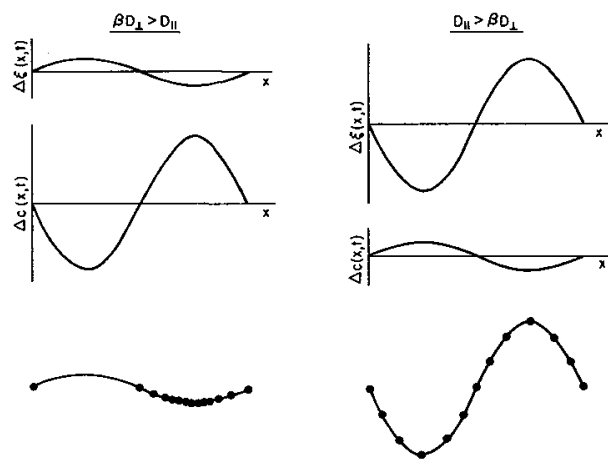

Fig. 2. Schematic representation of variation of boundary displacement $\Delta \xi(x, t)$ and concentration profile $\Delta c(x, t)$ from that of a planar boundary as a function of spatia1 coordinate $x$. (a) Diffusion of impurities perpendicular to the boundary is much greater than diffusion parallel $\left(D_{\downarrow}>D_{11}\right)$, and (b) diffusion of impurities parallel to the boundary is much greater than diffusion perpendicu$\operatorname{lar}\left(D_{\|}>D_{\perp}\right)$.

A useful summary of the aforementioned analysis is presented in Fig. 3, wherein macroscopic grain boundary velocity is plotted versus bulk impurity concentration for realistic values of material parameters. Three separate regions are evident: (1) a region labeled stable, wherein a planar grain boundary shape and a uniform lateral impurity profile are stable, so that results steming from the previously discussed models remain valid, (2) a region labeled unstable, wherein a planar grain boundary shape and a uniform lateral impurity profile are inherently unstable, so that results steming from the previously discussed models are not necessarily valid, and (3) a region labeled breakaway, wherein breakaway of a planar boundary from a uniform lateral concentration of impurities occurs. In general, a planar grain boundary characterized by a uniform lateral concentration profile of impurities is not expected to be stable throughout the usual range of experimental conditions.

Although results stemming from continuum planar-boundary models seem to differ greatly from the relaxed model, all theories tend to predict the same results; namely, that grain boundary migration may be divided into three fundamental regions: the low velocity region, the breakaway region, and the unstable region. The difference between these models is merely that in the unstable region a moving grain boundary assumes a variety of nonplanar configurations, characterized by nonuniform lateral impurtiy profiles, in anticipation of the breakaway process. The relaxed model predicts that the scale of such fluctuation generally is at the submicron 1evel, al- 


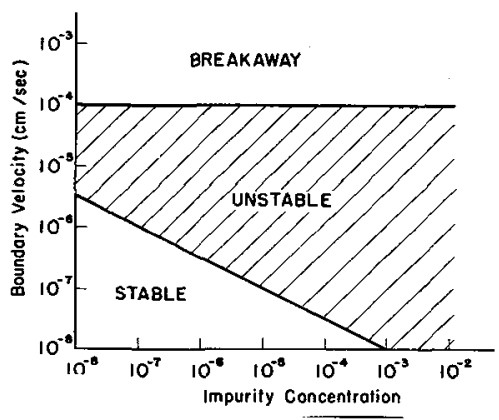

Fig. 3. Macroscopic grain boundary velocity $v_{0}$ versus excess impurity concentration $c_{o}$. Regions of planar boundary stability, instability, and breakaway are denoted for $D_{\perp}=D_{\|}$and typical values for material parameters. Hatched area indicates region wherein a planar grain boundary shape and a uniform lateral impurity concentration profile are unstable.

though it is difficult to estimate values of $D_{\perp}$ and $D_{n}$ accurately. Nevertheless, the magnitude of such events suggests that fundamental mechanisms for grain boundary migration may only be observable on a truly microscopic (submicron) scale.

Throughout the previous discussion it has been assumed that the retarding, or drag force is due to interaction of solute atoms with the moving grain boundary in such a way that $\mathrm{F}$ is proportional to $\mathrm{v}$. Other forms or types of drag force, however, are also possible. For example, Gleiter ${ }^{1}$ has suggested that the non-equilibrium generation of vacancies at the boundary gives rise to a velocity-dependent drag force, whereas Grabski 12 has suggested that differences between transferral rates for solvent and solute atoms from one grain to another can also give rise to a drag force, regardless of interactions of solute atoms with the grain boundary. Such refinements do not alter the general form of previous results but certainly do modify interpretation of the concomitant physical processes.

Perhaps a more severe criticism of the aforementioned models for grain boundary migration is the fact that grain boundaries are characterized by discrete and periodic atomic structure and, therefore, should not be represented by structureless continuum models. Because of the complexity of atomic structure, sensitive dependence on grain misorientation and boundary inclination, and necessity to focus on the behavior of individual boundaries at the highest possible level or resolution, little quantitative information is available, especially under controlled conditions of temperature, impurity content, defect structure and driving force. Nevertheless, with the advent of high-resolution electron optic techniques, momentum is shifting to the study of atomic mechanisms of grain boundary migration. 3,14 Some of these investigations are reviewed later in this article.

\section{Experimental Techniques}

Rates of grain boundary migration generally are extracted from measurements of recrystallization and/or grain growth. Such measurements, however, often lack a degree of control of important variables and usually are related to an "average" grain boundary structure, thus renouncing all possibility to assess the effect of boundary structure on grain boundary migration. A recent review of such experimental techniques and a critique of results is presented elsewhere. 4

More recent experimental techniques have focused on the role of grain boundary structure by utilizing bicrystalline specimens. Such techniques often rely on reduction of grain boundary area with displacement in order to provide the driving force for grain boundary migration. To exploit this particular phenomenon, special specimen configurations are required, some of which are illustrated schematically in Fig. 4. The wedge-shaped (direct capillary) configuration, illustrated in Fig. 4a, produces a simple driving force on an originally planar boundary (dashed line) which, in steady state, is given by the expression 


$$
F=\gamma / \dot{a},
$$

where $Y$ denotes grain boundary free energy and a denotes the displacement from the wedge apex along a vertical face. In this case, driving force increases with increasing grain boundary displacement. The reversed-capillary configuration illustrated in Fig. 4b, produces a driving force on an originally planar boundary (dashed line) intersecting a free surface at an acute angle $\alpha$, which, in steady state, is given by the expression

$$
F=\gamma f(\alpha) / a
$$

where $f(\alpha)$ denotes a dimensionless amplification factor which increases from unity at $\mathrm{d}=34^{\circ}$ to nearly 100 at $\alpha=2^{\circ} .15$ In this case, the initial driving force is very large (since a in $\mathrm{Eq} .8$ is of atomic dimensions) and decreases with increasing grain boundary displacement. It should be noted that the wedge-shaped configuration relies on the reversed-captllary effect for initial (non-uniform) displacement and, since $\alpha$ is nearly $90^{\circ}, f(d) \ll 1$ so that the boundary may never be able to overcome existing retarding forces.
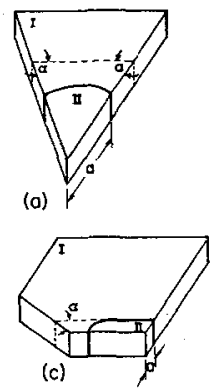
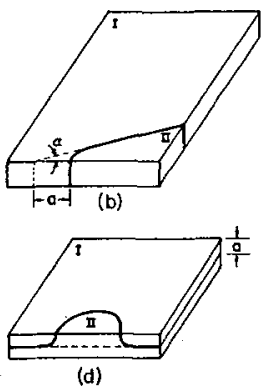

Fig. 4. Various bicrystalline specimens for study of grain boundary migration: (a) wedge technique $(F=\gamma / a)$, (b) reversed capillary technique $(F=\gamma f(\alpha) / a)$, (c) constant velocity technique wherein grain boundary is perpendicular to surface $(F=\gamma / a)$, and (d) constant velocity technique wherein grain boundary is parallel to surface $(F=\gamma / a)$. All techniques make use of capillarity to drive the boundary from the initial position (dashed line) to final position (solid line).

Whereas the wedge and reversed-capillary configurations involve non-uniform driving forces; $1 . e .$, a varies with grain boundary displacement, other configurations produce constant driving forces. The configuration illustrated in Fig. 4c, after an initial transcient stage, produces a constant driving force given by Eq. 7, but with a defined as the (constant) distance between the grain boundary and the (para1le1) free surface. Lastly, the sandwich-shaped configuration illustrated in Fig. 4d is oriented with the original grain boundary parallel to the specimen surface. After an initial transcient stage wherein the grain boundary breaks through to an external surface, a constant driving force, given once again by Eq. 7, is produced, where, in this case, a denotes specimen thickness, as defined in Fig. 4d. This particular technique is especially advantageous since extremely large values of the driving force can be produced in bicrystalline thin films and, in principle, resulting grain boundary migration can be monitored in situ on the hot stage of a transmission electron microscope.

of course, the simplified expressions for driving force derived from the aforementioned configurations are complicated by difficulty of specimen preparation, nonideal grain boundary shapes and jerky grain boundary motion, caused by grain substructure and a variety of microscopic pinning forces. Nevertheless, the aforementioned techniques represent a significant advance over eariier techniques and a portion of Sec. 4 is devoted to a survey of results steming therefrom. More complex grain boundary shapes are analyzed and further details on experimental techniques are presented elsewhere. 2,4 


\section{Recent Experimental Results}

The fact that grain boundary velocity varies linearly with driving force only. for sufficiently high purity or driving force has been emphasized in several experimental investigations. 12,16 In fact, the so-called driving force exponent $m$ (cf. Eq. 3) only approaches unity for very large driving forces; i.e., $\mathrm{F}>10^{6} \mathrm{erg} / \mathrm{cm}^{2}$. The results of Rath and $\mathrm{Hu}$ l, 18 most noticeably deviate from $m=1.0$, probably due to the fact that a wedge-shaped specimen configuration was employed, which results in characteristically low driving forces and great variation of results, depending on details of the experimental procedure. Regardless of the reasons, it is evident that extrinsic behavior dominates when the impurity content is too large or when the driving force is too small; i.e., when $F<$ the drag force $F_{c}$, where, in most analyses, $\mathrm{F}_{\mathrm{C}}$ is proportional to the impurity concentration.

In an attempt to increase the driving force (in order to circumvent the difficult problem of reducing the impurfty content below parts per million), techniques based on the specimen configuration illustrated in Fig. 4b have been applied to a variety of crystalline materials. One of the first applications was to NaCl bicrystals, characterized by various angles of misorientation $\theta$ about the common [001] axis, 19 wherein intrinsic grain boundary motion, characterized by an activation energy QI, which increases as $\theta$ decreases, is sustained by the driving force. For large $\theta, Q_{I}$ denotes the activation energy for self diffusion across the boundary. For small $\theta$, however, $Q_{I}$ nearly coincides with the activation energy for bulk self duffusion of the slower ionic species $\left(\mathrm{Cl}^{-}\right)$, indicating the occurrence of dislocation climb processes involving self diffusion through the perfect lattice. Moreoyer, an effective dislocation core radius, within which enhanced diffusion occurs, was estimated to be about $6 \AA$ from the transition between large and small $\theta$ behavior. Therefore, the difference in grain mobility between 10 w and high-angle boundaries is related to the structure of the boundary (or dislocations therein) itself.

The investigation of grain boundary migration by the reversed-capillary method has been extended to metals such as copper 20,21 and zinc. 22,23 . In the case of copper, grain boundary migration has been investigated for various. [001] tilt boundaries as a function of driving force, angle of misorientation and temperature. In a11 cases, grain boundary displacements obey a parabolic rate law and concomitant velocities are linearly related to driving force, implying that intrinsic grain boundary mobilities were measured. Again, migration of low-angle boundaries was characterized by an axtivation energy nearly identical to that for lattice self diffusion, whereas migration of random high-angle boundaries was controlled by direct atom transfer across the boundary. One interesting result was that the activation energy $Q$ for migration of a near coincidence boundary $\left(37 \pm 1^{\circ}\right)$ was determined to be larger than that for similar random high-angle boundaries. High mobilities occur however, even though $Q$ is large, due to the fact that the pre-exponential diffusion coefficient, $D$ is also. much larger. In contrast, Aust and Rutter 24 report values of $Q$ for coincidence boundaries in high purity lead which are about one-half of those for random boundaries and values of $\mathrm{D}_{\mathrm{o}}$ which are several orders of magnitude smaller. Higher mobility of coincidence boundaries was attributed to the fact that chemical impurities should not be as strongly attracted to the less-disordered structure of such boundaries. The differences between these results suggest that knowledge of the detailed structure of coincidence boundaries may be necessary in order to identify the true mechanisms of grain boundary migration.

Migration kinetics of special <110> tilt boundaries in $99.998 \mathrm{wt. \%}$ aluminum bicrystals have also been studied by the reversed-capillary method. 25 During grain boundary migration such boundaries assume complex faceted shapes and are characterized by relatively low mobilities, in fact the $70.5^{\circ}$ [110] boundary, corresponding to a (111) twin orientation, was totally immobile, even when annealed for extended periods of time just below the melting point. It was concluded that high-density [110] coincidence boundaries in nominally pure aluminum are characterized by lowmobility and that high coincidence density is not a sufficient condition for high macroscopic grain boundary mobility. In addition, observations suggest that facets are not microscopically planar but, rather, are characterized by a step structure, wherein motion of relatively low-mobility coherent twin boundaries (terraces) is 
facilitated by lateral motion of relatively high-mobility non-coherent twin boundaries (ledges). Other examples of faceting have been reported by Cosandey and Bauer. 26 More recently, similar behavior has been observed in situ by synchronton $x$-ray topography. 27 Results indicated that anisotropy of grain mobility is dependent on the number of steps resulting from the intersection of the boundary with $\{111\}$ planes and the defect structure of the growing grains.

The specimen configuration illustrated in Fig. $4 \mathrm{~d}$ recently has been used to investigate migration of [001] twist boundaries in bicrystalline thin films of silver by transmission electron microscopy. 28 Measured values of activation energy were consistent with those expected for grain boundary migration, but values do not seem to vary in a sensible manner with angle of misorientation, especially near low-angle and special high-angle grain boundaries. Either the kinetics of twist boundary migration are insensitive to boundary structure or such dependence was masked by other effects. Although more attention could have been devoted to the role of grain substructure and details of resulting boundary structure, this technique is certainly promising for correlation of grain boundary migration kinetics with high resolution of grain boundary structure, especially under conditions of exceptionally large driving forces.

In a more recent investigation of grain boundary migration in 99.99 wt.\% aluminum bicrystals, Fukutomi and Horiuchi 29 have utilized external stress as a driving force on symmetric grain boundaries. It was observed that the velocity of these boundaries could be expressed as

$$
v=A(\theta, \zeta) \sigma^{n} \exp (-Q / R T)
$$

where $A(\theta, \zeta)$ is a numerical factor depending on the angle of misorientation $\theta$ and the type of grain boundary dislocations, $\sigma$ is the applied stress and $n$ is an exponential factor determined to be about unity. Therefore, the velocity varies linearly with driving force $\theta \sigma$, although the dependence on $\theta$ was a function of the type of dislocation active in the migration process. In fact, $A(\theta, \zeta)$ actually decreased with increasing $\theta$, in contradiction to predicted behavior, indicating that the number of active dislocations is a complicated function of $\theta$. In addition, activation energies were obtained for the migration process as a function of $\theta$. As preyiously reported for $\mathrm{NaCl}$ and copper, the activation energy yaried from that close to lattice diffusion for low-angle boundaries to that for grain boundary diffusion for higher boundaries, with the transition occuring at about $5^{\circ}$. At angles of misorientation greater than about $25^{\circ}$ for [112] tilt boundaries and greater than about $10^{\circ}$ for [110] and [100] tilt boundaries, grain boundary sliding rather than grain boundary migration was observed. The results were interpreted in terms of lattice controlled diffusion between individual dislocations at low values of $\theta$, grain boundary controlled diffusion at intermediate values of $\theta$, and grain boundary sliding at high values of $\theta$, presumably because the stress can be relaxed more easily once the grain boundary assumes a more random structure. The results of this investigation clearly indicate the importance of lattice diffusion between individual dislocations at low angles of misorientation and a complicated dependence of grain boundary mobility on the type of active dislocation at larger angles of misorientation.

From the previous discussion, as well as from a more thorough review of recent publications, it is clear that evidence for the important role of grain boundary structure in determining concomitant migration behavior is mounting. For example, the results of Fukitomi and Horiuchi can only be explained by variations in grain structure, composed of various combinations of grain boundary dislocations. This particular topic has received considerable attention recently through careful observation of migration of special grain boundaries by transmission electron microscopy. 30,31 an important conclusion stemming from such investigacions is that grain boundary velocity may be expressed in terms of the motion of individual grain boundary dislocations, although a universal expression has not been fully developed. Nevertheless, increasing emphasis on correlation of grain boundary structure with migration kinetics should lead to significant advances in the understanding of grain boundary migration. 
another important issue which continues to receive attention. For example, it is generally observed that the low-velocity limit of the solute drag theories are in agreement with experimental results. ${ }^{2}, 32$ Recently, the effect of small amounts of silicon on the kinetics of grain boundary migration in copper has been investigated by Zordan and Bauer. ${ }^{33}$ It was observed that grain boundary displacements increased with increasing time and temperature and are characterized by an apparent activation energy consistent with that expected for grain boundary diffusion of silicon. Results are in excellent quantitative agreement with theories of grain boundary migration and indicate that the controlling mechanism is thermally activated transport of silicon across the moving boundary.

Recently, a phenomenon termed diffusion induced boundary migration (DIBM) has received considerable experimental and theoretical attention. This phenomenon involves grain boundary diffusion of one soluable component in another and subsequent mixing (alloying) by grain boundary migration, especially at temperatures where lattice diffusion is prohibitively slow. In this regard, DIBM is the reverse process of discontinuous precipitation. An example of DIBM is presented in Fig. 5 wherein a polycrystalline thin film of gold was coated with copper, annealed for $137 \mathrm{~h}$ at $150^{\circ} \mathrm{C}$, and examined by transmission electron microscopy after removal of excess copper from the external surfaces, thus revealing the initial $(x=0.4 \mu \mathrm{m})$ positions of a [111] tilt boundary. ${ }^{44}$ Several interesting features can be noted: First, the region in the wake of the moving boundary has been alloyed with copper, but in decreasing amounts with increasing displacement. Second, the original position of the boundary is outlined by a diffuse array of misfit dislocations necessary to accommodate the difference in lattice parameter between the alloyed and unalloyed regions. Lastly, the final positions of the boundary is outlined by a sharp (highangle) high-mobility grain boundary. It is basically agreed that DIBM can only occur when solute is supplied at temperatures sufficient for grain boundary diffusion but too low for appreciable lattice diffusion. What is not fully understood, however, is the fact that not all grain boundaries migrate under identical conditions, although it is believed that, once again, grain boundary structure playes a dominant role. Analyses of DIBM have been advanced by Balluffi and Cahn ${ }^{35}$ and Shewmon, 36 but, to date, grain boundary structure has not been adequately considered. Again, complete understanding of grain boundary migration must await careful correlation of boundary structure and migration kinetics.

(a)

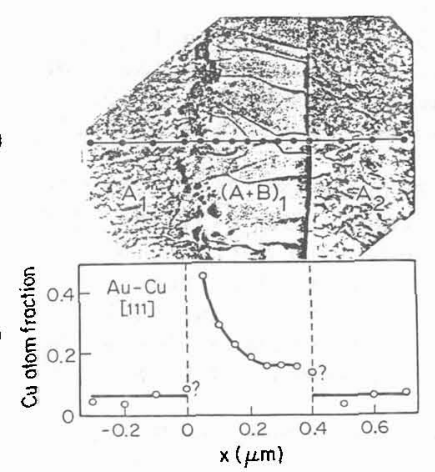

Fig. 5. Diffusion induced boundary migration in the gold layer of a [111] tilt boundary in a Cu/Au/Cu thin film sandwich following an anneal at $150^{\circ} \mathrm{C}$ for $137 \mathrm{~h}$. (a) Micrograph of the alloyed zone and (b) variation of composition within this zone. (After Pan and Balluffi. ${ }^{34}$ )

\section{Summary}

The purpose of this article has been to review recent theoretical and experimental advances in the understanding of grain boundary migration. Current theoretical understanding in pure materials is based on thermally activated transfer of single atoms or small clusters of atoms from one grain to another. In impure materials, however, grain boundary migration is affected by both the interaction of impurities with the moving boundary and the weighted thermally activated motion of both impurity 
and host atoms. The general behavior of such boundaries is well established, but details concerning grain boundary shape and impurity redistribution are not fully understood.

Current experimental advances are based on better control of driving force and grain boundary structure. The kinetics of grain boundary migration remain in general agreement with the aforementioned theoretical analyses. It is becoming more evident, however, that grain boundary migration is indeed affected even by small variations in grain boundary structure. Future directions certainly must address the nature of this structure, the concomitant distribution of impurities, and to what extent grain boundary migration is affected independently by these phenomena or collectively by some combination thereof. The advent of high-resolution instrumentation, coupled with more emphasis on control of boundary structure, should lead to important advances in the understanding of grain boundary migration in the foreseeable future.

\section{References}

1. S. Hofmann and F. Haessner in Recrystallization of Metallic Materials, F. Haessner, Ed., Dr. Riederer Verlag GmbH, Stuttgart (1970), p. 81.

2. C. J. Simpson, W. C. Winegard and K. T. Aust in Grain Boundary Structure and Properties, G. A. Chadwick and D. A. Smith, Eds., Academic Press, New York (1976).

3. H. Gleiter and B. Chalmers in Progress In Materials Science, B. Chalmers, J. W. Christian and T. B. Massalski, Eds., Pergamon Press, Oxford 16, 1 (1972).

4. M. S. Masteller and C. I. Bauer in Recrystallization of Metallic Materials, F. Haessner, Ed., Dr. Riederer Verlag GmbH, Stuttgart (1978), p. 251.

5. K. Lücke and K. Detert, Acta Met. 5, 628 (1957).

6. J. W. Cahn, Acta Met. 10, 789 (1962).

7. K. Luicke and H. P. Stüwe in Recovery and Recrystallization of Metals, H. Himmel, Ed., Interscience, New York (1963), p. 171 .

8. K. Lijcke and H. P. Stüwe, Acta. Met. 19, 1087 (1971).

9. A. Roy and C. L. Bauer, Acta Met. 23,957 (1975).

10. C. L. Bauer, J. Physique $36, C 4-333$ (1975).

11. H. Gleiter, Acta Met. 10,789 (1969).

12. M. W. Grabski, Po1. Akad. Nauk. Pr. Kom. Nauk. Techn. Met. 1063 (1969).

13. G. A. Chadwick and D. A. Smith, Eds., Grain Boundary Structure and Properties, Academic Press, New York (1976).

14. F. Cosandey and C. L. Bauer in Preparation and Properties of Thin Films, K. M. Tu and R. Rosenberg, Eds., Academic Press, New York (1982).

15. R. C. Sun and C. L. Bauer, Acta Met. 18, 635 (1970).

16. K. T. Aust and J. W. Rutter in U1tra-High Purity Metals, ASM, Cleveland (1962) p. 643-648.

17. B. B. Rath and H. Hu, Trans. AIME 245, 1243 (1969).

18. B. B. Rath and H. Hu, Trans. AIME $\underline{245}, 1577$ (1969).

19. R. C. Sun and C. L. Bauer, Acta Met. 18, 639 (1970).

20. R. Viswanathan and C. L. Bauer, Acta Met. 21, 1099 (1973).

21. R. Viswanathan and C. L. Bauer, Trans. AIME 5, 1691 (1974).

22. L. S. Shvindlerman and E. M. Fridman, Soviet Solid State Physics 15, 2466 (1974).

23. Ch. V. Kopetskii, V. G. Sursaeva and L. S. Shvindlerman, Fiz. Tverd. Tela 21, 401 (1979).

24. K. T. Aust and J. W. Rutter, Trans. AIME 215, 119 (1959).

25. M. S. Masteller and C. L. Bauer, Acta Met. 27, 483 (1979).

26. F. Cosandey and C. L. Bauer, Acta. Met. 28, 601 (1980).

27. J. Gastaldi and C. Jourdan, J. Crystal Growth 52, 949 (1981).

28. R. Pareja, Z. Metall. 72, 198 (1981).

29. H. Fukutomi and R. Horiuchi, Trans. Japan Inst. of Metals 22, 633 (1981).

30. C.M.F. Rae and D. A. Smith, Phil. Mag. A 41, 477 (1980).

31. C.M.F. Rae, Phi1. Mag. 44, 1395 (1981).

32. P. Gordon and R. A. Vandermeer in Recrystallization, Grain Growth and Textures, ASM, Cleveland (1966) p. 205.

33. R. D. Zordan and C. L. Bauer, Acta Met. 29, 1437 (1981).

34. J. D. Pan and R. W. Balluffi, Acta Met. $\underline{30}, 861$ (1982).

35. R. W. Balluffi and J. W. Cahn, Acta Met. 29, 493 (1981).

36. P. G. Shewmon, Acta Met. 29, 1567 (1981). 


\section{DISCUSSION}

L. HOBBS :

You have indicated that migration of pure boundaries can be adequately understood in terms of single atom jumps across the boundary. Are not cooperative effects observed? If not, why not?

C.L. BAUER : In most experiments, the single-atom tranfert theories are entirely adequate for the explanation of results. Namely, the measured activation energy corresponds to bulk diffusion activation energies for single atoms at low angles of misorientation and to grain boundary diffusion of single atoms at high angles of misorientation. Moreover, the pre-exponentiel terms include an entropy factor consistent with single-atom transfer. The one exception I know of is for certain special (coincidence) boundaries, where some cooperative motion may be necessary, such as rotation of rings of atoms within the coincidence site lattice unit cell, in order to affect displacement of the boundary .

P. LESBATS : It is a comment to say how migration study is a hard job. In most grain boundary migration measurements the grain boundary plane changes continuously. The interpretation supposes the migration of a grain boundary of a definite misorientation around a definite axis is independant on the direction of the normal to the grain boundary : this is not obvious.

C.L. BAUER : It is true that, in most "controlled" experiments on grain boundaxy migration, the grain boundary inclination varies even though the misorientation between grains remains fixed. Of course, this variation introduces some degree of uncertainty into such experiments, but, in my opinion, this uncertainty is a relatively small consideration. A more important effect is the frequent faceting of moving grain boundaries, especially on a scale too fine to resolve by conventional experimental techniques. 